\title{
Epidemiological Assessment of Contagious Bovine Pleuropneumonia in Central Tanzania
}

\author{
Mngumi $\mathrm{S}^{* 1}$, Makungu $\mathrm{S}^{2}$, and Mdetele $\mathrm{D}^{\mathbf{1}}$ \\ ${ }^{1}$ Zonal Veterinary Centre, P.O. Box 1752 Dodoma,Tanzania. \\ ${ }^{2}$ Ministry of Livestock and Fisheries P. O. Box 2870 Dodoma,Tanzania \\ *Corresponding author's Email: sbmngumi@yahoo.com; (DORCiD: 0000-0002-6263-7539
}

\begin{abstract}
A retrospective study was conducted to determine the epidemiology of Contagious Bovine Pleuropneumonia (CBPP) in the Central Zone of Tanzania. The present study used data from archived information of Central Zone Veterinary Centre (CZVC) for the past five years in the forms of weekly, monthly, and slaughterhouse reports, as well as Event Mobile Application (EMA-i) reports submitted to the zone. The present study found that out of 14 Local Government Authorities (LGAs) in the Central Zone, 10 reported the disease in the past five years. Moreover, 56, 426, and 11147 cases were reported as deaths, and the cattle at risk respectively. Therefore, $3.8 \%, 13 \%$, and $0.5 \%$ were reported as CBPP prevalence, case fatality rate, and mortality rate, respectively. It was also revealed that there was a clear temporal pattern of CBPP occurrence, with more cases being reported between August to December. In conclusion, CBPP was a seasonal problem in Central Tanzania. Therefore, the present research recommended the strengthening of control measures against this disease in the central zone of Tanzania. In order to be able to assess the actual burden of the disease on-site, this extensive study must be carried out, since the parameters obtained during the study were lower compared to the situation on-site.
\end{abstract}

Keywords: Central zone, Contagious bovine pleuropneumonia, Prevalence and distribution

\section{INTRODUCTION}

Contagious Bovine Pleuropneumonia (CBPP), is a significant constraint to cattle production throughout sub-Saharan Africa (Wakgari et al., 2018). The causative agent of CBPP is Mycoplasma mycoides subspecies mycoides (small colony), which is transmitted through direct contact. Clinically, CBPP is characterized by three forms, including preacute, acute, and chronic forms. Temporary, obvious infections and the presence of persistently infected animals with the encapsulated infected sequestra are features of chronic CBPP that promote further spread of the disease (Di Provvido et al., 2017). In 2015, 45 Local Government Authorities (LGAs) reported CBPP in mainland Tanzania, in which a total of 3795 cattle were infected and 706 of them died. Since 2013, the government has spent 1,835,166,910.00 Tanzanian shillings (over USD 1,000,000) to purchase 15,185,800 doses of CBPP vaccines, resulting in national and economic losses (Matthew et al., 2016). The present study aimed to describe the status and seasonality of CBPP in the Central Zone of Tanzania, and necessary information is needed to combat the disease in the zone and the country in general.

\section{MATERIALS AND METHODS}

\section{Ethical approval}

The present research has been conducted in accordance with ethical principles issued by the Ministry of Livestock and Fisheries in Tanzania, under the Animal Welfare Act (No. 19, 2008).

\section{Study area}

The study was conducted in central Tanzania, the zone that consisted of two administrative regions, Dodoma and Singida, which have 14 LGAs. The geographical coverage of the zone was approximately $48653.3 \mathrm{~km}^{2}$ (Dodoma 41,310 $\mathrm{km}^{2}$ and Singida 7,343.3 $\mathrm{km}^{2}$ ) with a cattle population of 3.6 Million (Figure 1).

\section{Study design}

In this retrospective study design, archived data from CZVC, which were collected and used in the study in the last five years (From January 2014 to December 2018). Weekly, monthly, abattoir, and Event Mobile Application (EMA-i) reports were used. The key variables retrieved were case reports, the population at risk, death reports, region, LGA name, village names, geo-coordinates, and observation date. 


\section{Data analysis and management}

The data retrieved were entered and managed in Microsoft Excel sheets, and checked for completeness before analysis (Data cleaning). Descriptive and analytical statistics were done using Microsoft Excel and Quantum GIS software, which were used for mapping using recorded coordinates of LGAs with which CBPP was reported.

\section{Prevalence, mortality and case fatality analysis}

The prevalence of CBPP was calculated using the Prevalence formula $(P: n / / N)$, where $P$ is the prevalence, $n$ is the number of sick animals in a specific place and at a specific time, and $\mathrm{N}$ is the population at risk at the same time (Joffe et al., 2011). Mortality (crude) was calculated by dividing the number of cattle that died during the referenced period by the population at risk over the same period (Chaudhary et al., 2013). Case fatality was estimated by dividing the number of cattle died from CBPP by the number of cases (Ghani et al., 2005).

\section{Map of Tanzania Showing Districts}
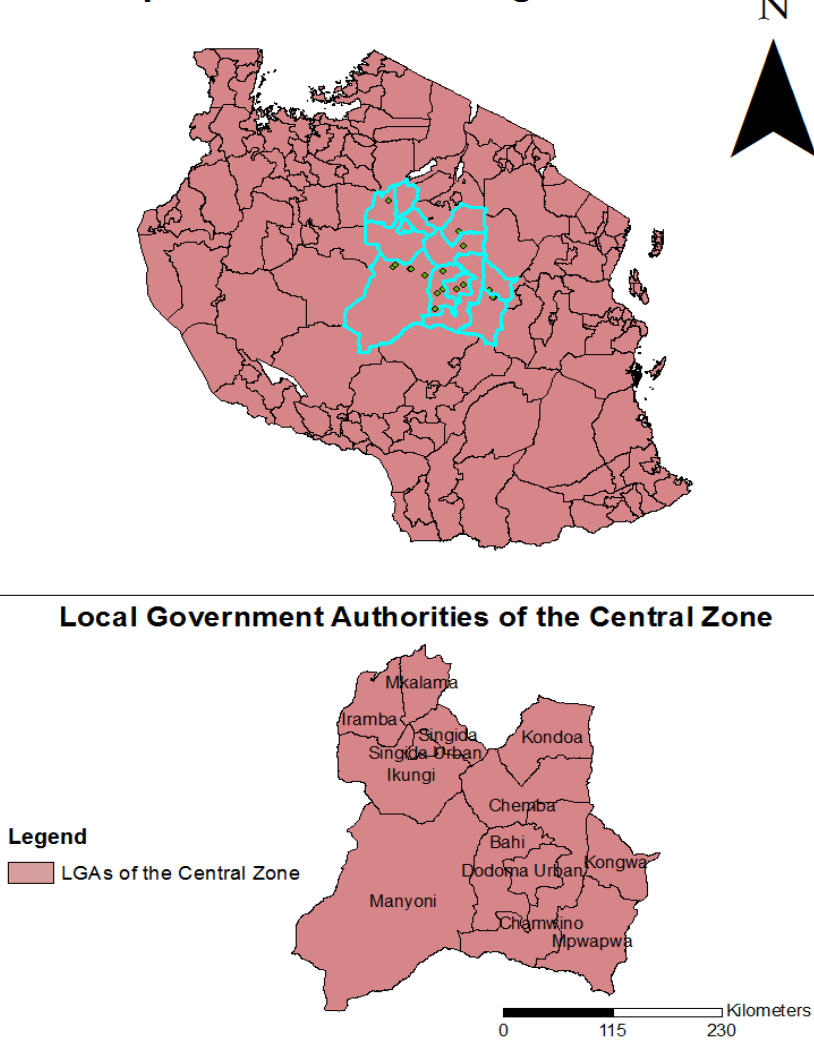

Figure 1. Districts of the study area in Tanzania consisted of two administrative regions (Dodoma and Singida)

\section{RESULTS}

During the five-year period from 2014 to 2018, a total of 426 CBPP cases and 56 deaths were reported in both regions. There was a variable number of CBPP cases each year. A total of 56 deaths due to CBPP were reported during the same period. Table 1 indicates that the maximum number of cases per year was 128, and the minimum was 23 cases.

\section{Prevalence of contagious bovine pleoropneumonia}

In total of 10 out of 14 LGAs reported the disease in the past five years. The overall prevalence of CBPP was $3.8 \%$ (426/11147). In contrast, mortality was $0.5 \%$ (56/11147), while case fatality was $13 \%(56 / 426)$. The number of affected districts, cases, deaths and population at risk for each year from 2014 to2018 is shown in Table 1.

\section{Mortality and case fatality from contagious bovine pleuropneumonia}

The mortality rate of CBPP was $0.5 \%$ (56/11147) reported from eight districts, while two districts reported no mortality despite reporting CBPP cases (Table 1). The case fatality of the disease was estimated at 13\% (56/426).

\section{Distribution of contagious bovine pleuropneumonia in central Tanzania}

The temporal and spatial pattern of the CBPP over the five years is shown in Figure 2 andFigure 3. The disease appeared to be distributed in many parts of the zone with the exception of Singida urban, Mkalama, and Ikungi. Further outbreaks were observed between August and December. 


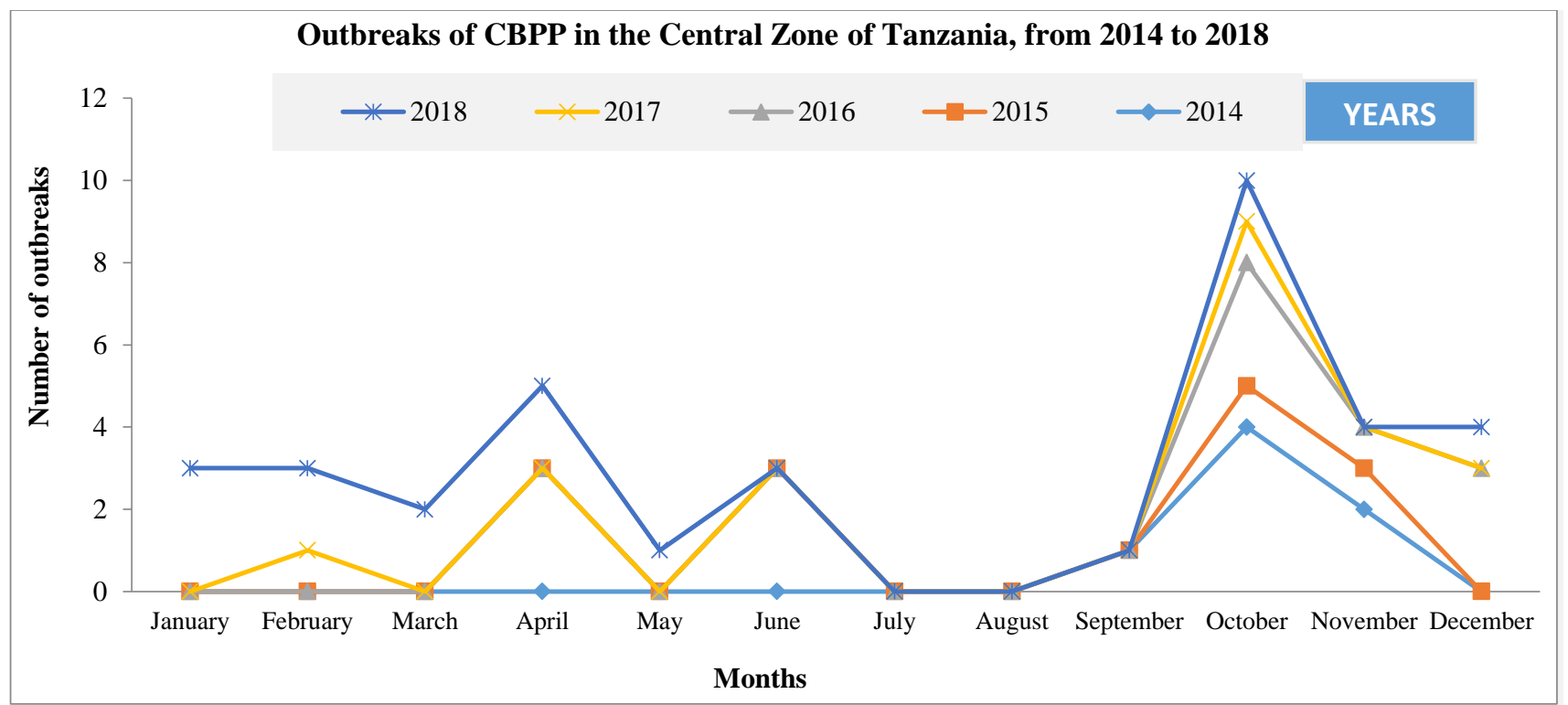

Figure 2. Time distribution of contagious bovine pleuropneumonia cases reported in Central Tanzania from 2014 to 2018

\section{Distribution of CBPP in the Central zone 2014-2018}

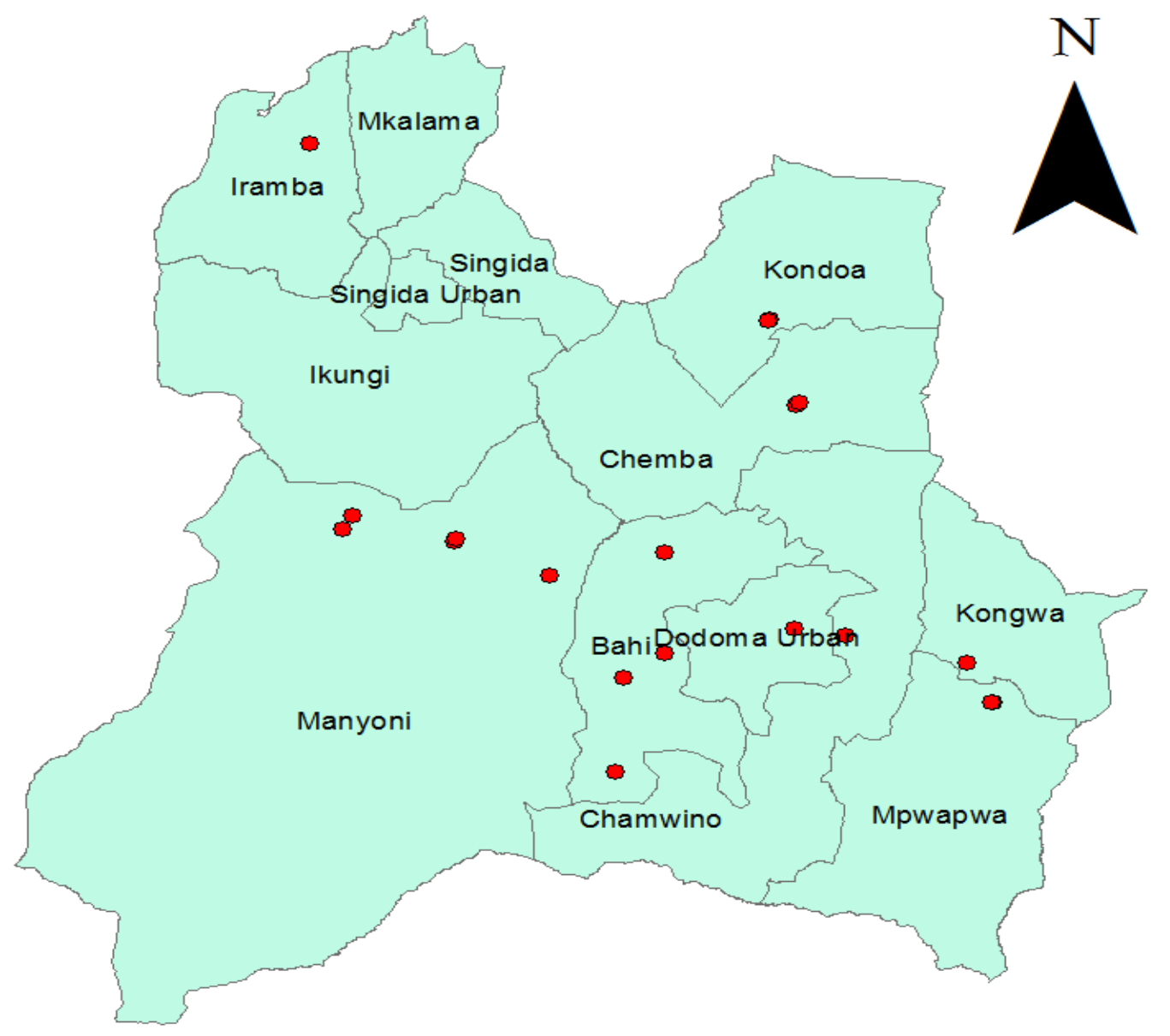

\section{Legend}

- Cases of CBPP LGAs of the Central Zone

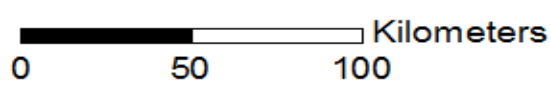

Figure 3. Spatial distribution of contagious bovine pleuropneumonia cases in central Tanzania from 2014 to 2018 
Table 1. The number of contagious bovine pleuropneumonia cases and deaths reported in Central Tanzania from 2014 to 2018

\begin{tabular}{lcccc}
\hline Year & Affected districts & Population at risk & Cases (\%) & Deaths $(\%)$ \\
\hline 2014 & 4 & 1646 & $113(6.8)$ & $22(1.3)$ \\
2015 & 4 & 748 & $100(13)$ & $10(1.3)$ \\
2016 & 6 & 2508 & $62(2.5)$ & $13(0.5)$ \\
2017 & 2 & 2454 & $23(0.9)$ & $2(0.1)$ \\
2018 & 5 & 3791 & $128(3.4)$ & $9(0.2)$ \\
Total & & 11147 & $426(3.8)$ & $56(0.5)$ \\
\hline
\end{tabular}

Table 1. Distribution of contagious bovine pleuropneumonia cases in districts of the Central Tanzania from 2014 to 2018

\begin{tabular}{lccc}
\hline District & Population at risk & Cases $(\%)$ & Deaths $(\%)$ \\
\hline Bahi & 358 & $68(19)$ & $10(2.8)$ \\
Chamwino & 105 & $14(13)$ & $3(2.9)$ \\
Chemba & 288 & $35(12)$ & $3(1)$ \\
Dodoma urban & 211 & $3(1.4)$ & $1(0.5)$ \\
Kondoa & 915 & $19(2.1)$ & $0(0)$ \\
Kongwa & 104 & $5(4.8)$ & $1(1)$ \\
Mpwapwa & 7463 & $184(2.5)$ & $9(0.1)$ \\
Iramba & 19 & $1(5.3)$ & $0(0)$ \\
Itigi & 183 & $7(3.8)$ & $1(0.5)$ \\
Manyoni & 1501 & $90(6)$ & $28(1.9)$ \\
Total & 11147 & $426(3.8)$ & $56(0.5)$ \\
\hline
\end{tabular}

\section{DISCUSSION}

The overall prevalence, mortality, and case fatality of CBPP in the present study was $3.8 \%, 0.5 \%$, and $13 \%$, respectively, which were lower compared to other studies. In a fully susceptible population, morbidity and mortality due to CBPP are always higher (Abdela and Yune, 2017). This low prevalence, mortality, and case fatality rate could be due to the endemicity of the disease or under-reporting of LGAs. Hence, few CBPP cases and deaths were reported to the central zone office. The present study demonstrated that a total of ten LGAs reported CBPP in the central Tanzania in the past five years, including Iramba, Itigi, Manyoni, Bahi, Chamwino, Kondoa, Kongwa, Mpwapwa, Chemba, and Dodoma. On the other hand, the remaining four LGAs of central zone did not report this disease for the entire period of the past five years; including Singida Municipal, Singida rural, Mkalama, and Ikungi. The possible explanation for some LGAs reporting CBPP while others didnot, could be that some LGAs with good technical veterinary professionals were effective compared to others, that they had good reporting tendencies than others. Therefore, it seemed like some LGAs have more CBPP cases than others. These differences in CBPP cases between LGAs in Central Tanzania could also be due to understaffing or inability of farmers to recognize CBPP cases which were compounded by syndromic nature of this disease, as many cases go unnoticed (Swai et al., 2013). The results indicated that there was a clear temporal pattern of CBPP outbreaks in the central Tanzania, with more CBPP outbreaks reported between August and December compared to other seasons (Figure 2). These results were in consistent with the results of a study conducted in Uganda in 2017, in which most CBPP cases were reported during November (Ssemadaali et al., 2017). The reason could be that at that time of the year, due to droughts that cause pasture and water shortages, animals gather in areas where it is possible to keep pasture and water. The close contacts between healthy and carrier animals in these areas enhanced the transmission of infection. It is worth noting that market points and pasture sharing contributed to animal contact and facilitate the transmission of the disease.

\section{CONCLUSION}

The study determined the prevalence of $4 \%$ for CBPP in central Tanzania based on passive surveillance at local government authority level in central Tanzania. The onset of the disease was also found to have a specific temporal pattern, revealing that duration of August to December was the peak time for CBPP in the central zone. Since CBPP remains a problem in central Tanzania, control measures for this disease across entire central Tanzania have to be strengthened, including vaccination and transmission control. Serological and meat inspection report studies will be much more important to get comprehensive results with laboratory-confirmed disease results for a proper evaluation of the disease status in the study area. 


\section{DECLARATIONS}

\section{Authors' contribution}

Makungu Selemani designed the study, Samwel Mngumi played a role in study design, data retrieval and analysis, and manuscript writing, Daniel Mdetele contributed in manuscript writing. Finally, all authors revised the manuscript and approved it for publication.

\section{Competing interests}

The authors declared that they have no competing interests.

\section{Acknowledgements}

This study was supported by Food and Agriculture Organization of the United Nations (FAO) including FAOTanzania, the Institute for Infectious Animal Diseases (IIAD), and Texas A\&M University through In-Service Applied Veterinary Epidemiology Training (ISAVET) Programme. The authors would also like to thank The Ministry of Livestock and Fisheries of Tanzania, through the Directorate of Veterinary Services, for authorizing this research. Great thanks are extended to Professor Esron Karimuribo, Dr. Emmanuel Swai, Dr. Erick Komba and Dr.Benezeth Lutege for their guidance. Special thanks go to the District Veterinary and Livestock Field Officers in Central Tanzania for reporting disease conditions to the Central Zone.

\section{REFERENCES}

Abdela N and Yune N (2017). Seroprevalence and distribution of contagious bovine pleuropneumonia in Ethiopia: Update and Critical Analysis of 20 Years (1996-2016) Reports. Frontiers in veterinary science, 4: 100. DOI: https://www.doi.org/10.3389/fvets.2017.00100

Chaudhary JK, Singh B, Shiv P and Verma MR (2013). Analysis of morbidity and mortality rates in bovine in Himachal Pradesh. Veterinary World, 6(9): 614-619. Availabe at: http://www.veterinaryworld.org/Vol.6/Sept-2013/6.pdf

Di Provvido A, Di Teodoro G, Muuka G, Marruchella G and Scacchia M (2017). Lung lesion score system in cattle: proposal for contagious bovine pleuropneumonia. Tropical Animal Health and Production, 50: 223-228. DOI: https://www.doi.org/10.1007/s11250-017-1409-2

Ghani AC, Donnelly CA, Cox DR, Griffin JT, Fraser C, Lam TH Ho LM, Chan WS, Anderson RM, Hedley AJ et al. (2005). Methods for estimating the case fatality ratio for a novel, emerging infectious disease. American Journal of Epidemiology, 162(5): 479486. DOI: https://www.doi.org/10.1093/aje/kwi230

Joffe D, Van Niekerk D, Gagne F, Gilleard J, Kutz S and Lobingier R (2011). The prevalence of intestinal parasites in dogs and cats in Calgary, Alberta. Canadian Veterinary Journal-Revue Veterinaire Canadienne, 52(12):1323-1328.Availabe at:: https://www.ncbi.nlm.nih.gov/pmc/articles/PMC3215466/

Matthew M, Mruttu H and Gebru G (2016). Animal health strategy and vision forTanzania. Available at: https://www.semanticscholar.org/paper/Animal-health-strategy-and-vision-for-Tanzania-MatthewMruttu/a21c6fc3ea91a7cb12f06e7580e85a2f8e3066d5

Ssemadaali MA, Majalija S, Mwebe R, Olet S and Khaitsa ML (2017). Spatial and temporal distribution of contagious bovine pleuropneumonia in Uganda (1956-2011). Pan African Medical Journal (ARTISSUE). Availabe at:: https://www.panafrican-medjournal.com/content/series/27/4/2/full/

Swai E, Mwezimpya I, Ulicky E, Mbise A and Moshy W (2013). An abattoir survey of contagious bovine pleuropneumonia lesions in slaughtered cattle in selected districts in Northern Tanzania. Asian Pacific Journal of Tropical Biomedicine, 3(4): 303-306. DOI: https://www.doi.org/10.1016/S2221-1691(13)60067-2

Wakgari M, Kitila G, Chali I, Merdasa D, Guta D, Gezahegn E and Kebede B (2018). Sero-prevalence of contagious bovine pleuropneumonia (CBPP) and its associated potential risk factors in selected districts of east Wollega zone, Oromia region, Ethiopia Austin Journal of VeterinaryScience andAnimal Husbandry, 5(2): id1043. Availabe at: https://austinpublishinggroup.com/veterinary-science-research/fulltext/avsah-v5-id1043.php 